\title{
HIV-1-cellular interactions analyzed by single virus tracing
}

\author{
Thomas Endreß · Marko Lampe · John A. G. Briggs • \\ Hans-Georg Kräusslich · Christoph Bräuchle • \\ Barbara Müller · Don C. Lamb
}

Received: 12 December 2007/Revised: 19 March 2008/Accepted: 19 March 2008/Published online: 10 April 2008

(C) The Author(s) 2008

\begin{abstract}
Single virus tracing (SVT) allows the direct investigation of the entry pathway of viruses into living cells. Using fluorescently labeled virus-like particles (VLPs) and SVT, we have studied the interaction between human immunodeficiency virus type 1 (HIV-1) and the plasma membrane of living cells. From the trajectories of freely diffusing VLPs in solution, we established that the particle preparation was homogeneous and the particles had a hydrodynamic radius of $86 \pm 5 \mathrm{~nm}$, consistent with the size of single HI viruses. The VLPs that come in contact with the cell surface either become immobilized or rapidly dissociate from the cell surface. The fraction of virions that become immobilized on the plasma membrane correlates with the surface heparan sulfate linked proteoglycans (HSPG) concentration of the cell line tested. The particles that are not immobilized make an average of 1.5 contacts with the cell surface before diffusing away. For
\end{abstract}

T. Endreß · J. A. G. Briggs · C. Bräuchle · B. Müller ·

D. C. Lamb $(\bowtie)$

Physical Chemistry, Department of Chemistry and Biochemistry, Ludwig-Maximilians-Universität München, Butenandtstr. 11,

Haus E, 81377 Munich, Germany

e-mail: don.lamb@cup.uni-muenchen.de

M. Lampe · H.-G. Kräusslich

Abteilung Virologie, Universitätsklinikum Heidelberg,

Im Neuenheimer Feld 324, 69120 Heidelberg, Germany

C. Bräuchle · D. C. Lamb

Center for NanoScience, Ludwig-Maximilians-Universität

München, Geschwister-Scholl-Platz 1, 80539 Munich, Germany

D. C. Lamb

Department of Physics, University of Illinois

at Urbana-Champaign, Urbana, IL 61801, USA most cell lines investigated, the contact duration follows an exponential distribution with a lifetime between 20 and $50 \mathrm{~ms}$ depending on the cell type.

Keywords HIV-1 - Heparan sulfate proteoglycans . Single virus tracing . Fluorescence microscopy

\section{Introduction}

The enveloped virus HIV-1 enters its host cell by fusion with a cellular membrane. The fusion process involves interaction of the viral envelope (Env) surface glycoprotein gp120 with the cellular receptor CD4, which triggers a conformational rearrangement that allows the binding of gp120 to a co-receptor molecule, usually CXCR4 or CCR5. Co-receptor recognition then leads to conformational changes within the viral transmembrane glycoprotein gp41, which mediates membrane fusion. While productive HIV-1 entry through direct fusion of the virion with the plasma membrane has been well established, we have recently shown that endocytotic uptake of HIV-1 can also contribute to productive infection (Daecke et al. 2005; Fackler and Peterlin 2000; Maurin et al. 2007). It is difficult to differentiate between different parallel viral-entry pathways or to dissect subsequent steps of the entry process using bulk biochemical and virological analyses, which have been widely used to investigate the HIV-1 entry process. Furthermore, although much has been learned about the interaction of HI virions with cells using these traditional approaches, little information is available regarding the dynamics of HIV-1 entry. Such information is important both for a fundamental understanding of the viral life cycle as well as for clarifying the function of entry inhibitors. 
Single virus tracing (SVT) uses ultra-sensitive wide-field fluorescence microscopy to track individual virus particles in real time with high spatial resolution and single molecule sensitivity (Bräuchle et al. 2002; Seisenberger et al. 2001). As the fluorescence is emitted by a single, sub-diffraction limited particle, the position of the particle can be determined to an accuracy of $<40 \mathrm{~nm}$ from the peak position of the observed fluorescent spot. With this technique, the individual steps of the entry process and the dynamics of these processes can be elucidated. SVT has been utilized to investigate the entry and trafficking of several viruses including adeno-associated virus (Bräuchle et al. 2002; Seisenberger et al. 2001), adenovirus (Suomalainen et al. 1999), simian virus 40 (Damm et al. 2005), murine polyoma virus (Ewers et al. 2005), HIV (McDonald et al. 2002), influenza virus (Lakadamyali et al. 2003; Rust et al. 2004), rabies virus (Finke et al. 2004) and murine leukemia virus (Lehmann et al. 2005). From the trajectories of individual viruses, the interactions between these particles and different cellular components can be directly observed.

For a detailed investigation of HIV-cell interactions by SVT, we have generated and characterized entry-competent single- as well as double-fluorescently labeled HIV-1 derivatives (Lampe et al. 2007; Müller et al. 2004). The dual-labeled virus like particles (VLPs) carry two differently colored fluorescent labels at two viral proteins and were designed to distinguish between complete virions and subviral particles resulting from membrane fusion. In our initial live-cell imaging studies using these VLPs, we had observed different types of particle behavior: free diffusion, brief touches with the plasma membrane, as well as a significant percentage of particles that displayed a rapid, presumably non-productive, immobilization at the plasma membrane in an apparently heparan sulfate dependent manner (Lampe et al. 2007). Heparan sulfate has been reported to be involved in HIV-1 cell attachment and to be of functional importance in some types of host cells, but the overall role of heparan sulfate for HIV-1 infection is not entirely clear (Bobardt et al. 2003; Moulard et al. 2000; Parren et al. 1998; Ugolini et al. 1999; Zhang et al. 2002). In the continuative study presented here, we had made use of the strengths of SVT to investigate the interactions of HIV-1 occurring at the plasma membrane in a quantitative manner.

\section{Materials and methods}

\section{Preparation of labeled VLPs}

293 T cells cultivated in DMEM with $10 \%$ fetal calf serum and antibiotics were transfected with a mixture of plasmids; pCHIV, pCHIV.eGFP and pmRFP-Vpr (Lampe et al.
2007 ) in a molar ratio of $1: 1: 0.8$ by calcium phosphate precipitation. At $44 \mathrm{~h}$ after transfection, medium was harvested and cleared, (Lampe et al. 2007) and VLPs were concentrated by ultracentrifugation through a $20 \%(\mathrm{w} / \mathrm{w})$ sucrose cushion. Particles were resuspended in ice cold PBS, $10 \%$ fetal calf serum and frozen. The particle concentration was quantified by p24 ELISA.

Flow cytometry analysis

Fluorescence labeling of cell surface molecules was performed using either allophycocyanin conjugated anti-CD4 antibody (BD Pharmingen) or fluorescein isothiocyanate labeled anti-heparan sulfate antibody (Seikagaku). Cells were harvested in PBS containing $0.7 \mathrm{mM}$ EDTA, washed and incubated with labeled antibody for $30 \mathrm{~min}$ at $4^{\circ} \mathrm{C}$ followed by analysis on a FACSCalibur using CellQuestPro software (BD Biosciences). Virus binding assays were performed using labeled particles (60 ng p24 per $10^{6}$ cells) instead of antibodies. Cells were incubated with VLPs for $30 \mathrm{~min}$ on ice, washed and subjected to flow cytometry analysis. Bound virus was quantified by determining eGFP mean fluorescence. For heparinase treatment, cells were incubated with $2 \mathrm{U}$ of heparinase I (Sigma) in $100 \mu \mathrm{l} \mathrm{PBS}$ for $30 \mathrm{~min}$ at $37^{\circ} \mathrm{C}$ and washed twice with ice cold PBS before virus addition. The efficiency of heparinase treatment was controlled by flow cytometry analysis.

\section{Multi-color single-virus tracing setup}

Measurements were performed on a wide-field fluorescence microscope (NIKON Te300) with single-molecule sensitivity (Bräuchle et al. 2002; Seisenberger et al. 2001). A schematic of the setup is shown in Fig. 1a. HeLa cells, HeLaP4 cells [carrying CD4 and co-receptors; (Charneau et al. 1994)] or CD4-positive or negative CHO cell derivatives (Zhang et al. 2002) were incubated with VLPs in $\mathrm{CO}_{2}$ independent medium (Invitrogen) at $37^{\circ} \mathrm{C}$ and illuminated in a wide-field geometry using both $476 \mathrm{~nm}$ from an argon-ion laser (Coherent Inc., Sabre Innova) and $532 \mathrm{~nm}$ light from a continuous-wave, frequency-doubled Nd:YAG laser (Soliton GmbH, DLSOS). The excitation beams were overlapped with a dichroic mirror (AHF Analysentechnik Q570LP). A programmable, mechanical shutter was placed in the excitation beam path and used to minimize sample exposure during time-lapse experiments. After the shutter, the excitation beam was expanded by a factor of 5 (Exp, Beam expander, Thorlabs) and the inner portion of the excitation beam is selected by a variable aperture. The light is then focused by an achromatic lens (Thorlabs) on the back focal plane of the objective (Nikon oil immersion $100 \times$ DIC N.A. 1.4). This provided us with a homogeneous, parallel illumination of the sample with a 

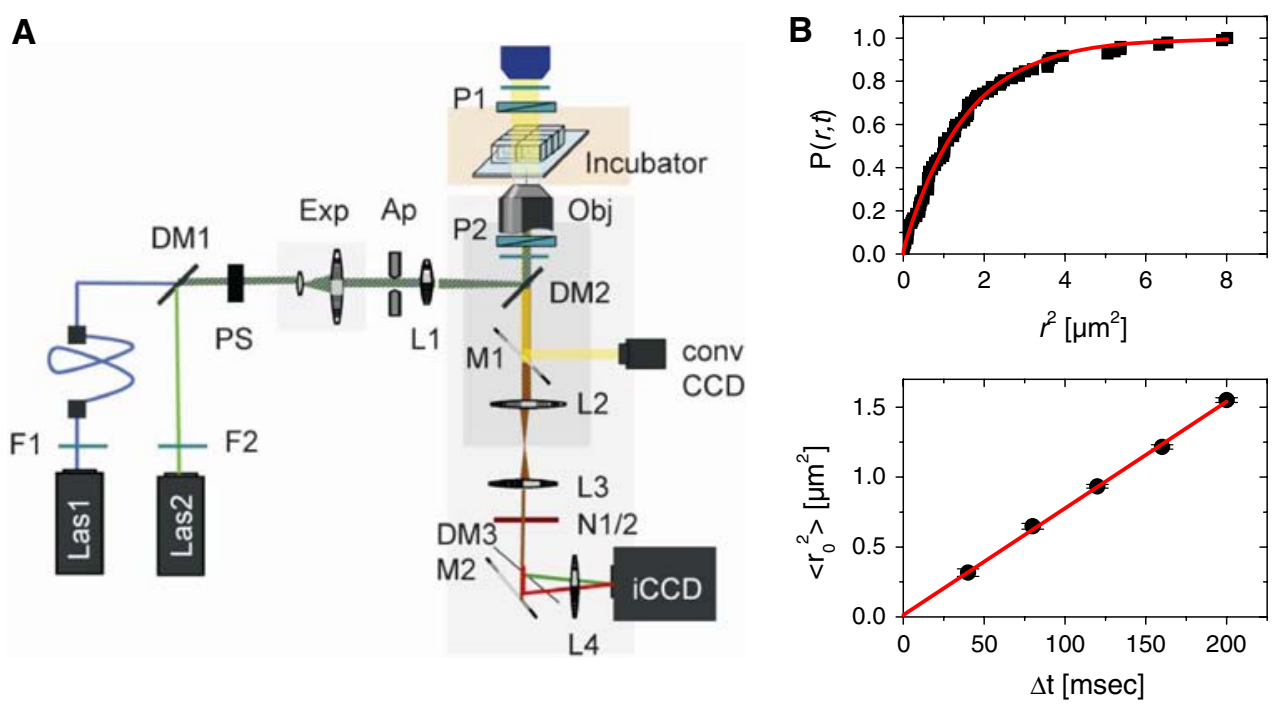

Fig. 1 a Schematic of the Single Virus Tracing apparatus. Las1 Argon-ion laser (476 nm), Las2 frequency-doubled Nd:YAG laser $(532 \mathrm{~nm}), F 1 / F 2$ laser clean up filters at 476 and $532 \mathrm{~nm}$ respectively, $D M$ dichroic mirrors, $M$ aluminum mirrors, $P S$ programmable shutter system, Exp $5 \times$ beam expander, $A p$ aperture, $L$ lenses, $L 1$ $f=250 \mathrm{~mm}, L 2 f=200 \mathrm{~mm}$, Nikon tubus lens, $L 3 f=100 \mathrm{~mm}$; L4 $f=150 \mathrm{~mm} ; \quad P 1 / P 2$ Wollaston prisms, Obj oil immersion objective $\times 100$ DIC N.A. 1.4, Incubator incubator chamber and objective heater, Conv. CCD conventional CCD camera, iCCD intensified CCD camera, N1/2 notch filters. b Characterization of labeled VLPs. (upper panel) Determination of particle size by diffusion measurements. The accumulated probability of the squared displacement was determined from single-molecule trajectories (symbols). A fit to Eq. (1) verified that the sample consisted of a single, homogeneous particle size (lower panel). An exemplary MSD plot as a function of lag time, $\Delta t$. The linearity of the plot indicates free diffusion and the slope yields a diffusion coefficient. From multiple experiments, an average diffusion coefficient of $D=1.78 \pm 0.11 \mu \mathrm{m}^{2} / \mathrm{s}$ was determined, corresponding to a hydrodynamic radius of $R_{\text {hyd }}=86 \pm 5 \mathrm{~nm}$ for single VLPs wide-field illumination diameter of approximately 25 microns.

The emitted fluorescence from particles in the illumination path was collected by the same objective, passed through the dichroic beamsplitter (AHF Analysentechnik, F53-007) and focused through the tube lens. The image plane is further relayed by two lenses onto the intensified charged coupling device (iCCD, I-PentaMAX, Princeton Instruments). Between the last two lenses, a dichroic beamsplitter (AHF Analysentechnik 580 DCXR) and mirror were used for spectral separation the eGFP and mRFP1 signals and were imaged on different regions of the iCCD camera. Two notch filters (N1 Kaiser \#2402 and N2, Heliopa Nr. 155 E72/52) were placed before the last beamsplitter to eliminate detection of scattered excitation light. For accurate overlapping of the eGFP and mRFP1 signals, a mapping between the two regions-of-interests on the iCCD camera was performed by imaging a portion of a $\mathrm{CD}$. The fingerprint of the $\mathrm{CD}$ provides a unique pattern that can be detected in both channels. The shift was determined by maximizing the overlap of the pattern of the $\mathrm{CD}$.

A microscope mounted conventional CCD camera (conv CCD) was used for collection of white light images of the cells. In addition to bright-field imaging, it was possible to record phase contrast or differential interference contrast, DIC, images (Nikon Wollaston prisms MEH48900,
MBH74295). All the measurements were performed at $37^{\circ} \mathrm{C}$ with the aid of an incubator chamber and an objective heater (Ink, Pecon).

Individual recordings were collected with a temporal resolution of $40 \mathrm{~ms}$ per frame over $16 \mathrm{~s}$. Under these conditions, photobleaching was observable over the course of the measurement. However, as the VLPs are labeled with several fluorophores, photobleaching causes a decrease in the fluorescence intensity of the VLPs during the movie but does not cause them to disappear as would be the case for single fluorophores. Hence, photobleaching does not alter the analysis or the results presented here. Spatial resolution was diffraction limited (typically $225 \mathrm{~nm}$ ) with an accuracy of $40 \mathrm{~nm}$ as determined from the fit of the fluorescence intensity distribution of a single particle to a two-dimensional Gaussian point-spread-function (Schmidt et al. 1995). For some experiments, single cells were imaged over $2 \mathrm{~h}$ for which we used time-lapse imaging. We measured a series of short time intervals with high time resolution interrupted by pauses in which the sample was not illuminated. This setup yields a time resolution of $40 \mathrm{~ms}$ during the short observation periods while allowing events occurring in a single cell to be followed over hours. From the high time-resolution portion of the movies, we could extract information about the diffusional behavior of the particle and, from the time-lapse images, observe slower processes. 
The TTL output of the ICCD camera was programmed to automatically open the excitation shutter when the camera was recording images. Control of the ICCD camera was performed through WinView software via an in-house programmed visual basic script.

\section{Data analysis}

The first step in data analysis was determination of the trajectories of individual VLPs. The data were analyzed using Metamorph (Universal Imaging Corp.) and Labview (National Instruments Corp.). Each frame was loaded and the number of the individual particle and its approximate position entered into the program. Improved spatial accuracy could then be determined by fitting the position of the individual spot to a two-dimensional Gaussian. The accuracy is dependent on the signal quality and was typically on the order of $40 \mathrm{~nm}$. The $x-y$ coordinates of the pixels were converted into nanometers using the calibration factor of $156 \times 156 \mathrm{~nm}^{2}$ per pixel.

Contacts between the VLP and the plasma membrane were determined using the following two criteria: a contact event was recorded when the VLP came within 1 pixel $(156 \mathrm{~nm})$ of the projection of the cellular membrane and a change in direction of the particle's motion continued over a distance of longer than 6 pixels $(936 \mathrm{~nm})$ was observed. A particle was considered to be immobile in the plasma membrane when the position of the particle did not move outside a radius of $R=2$ pixels $(312 \mathrm{~nm})$ between the initial contact of the VLP with the cellular surface and the end of the observation period.

\section{Results and discussion}

\section{Characterization of labeled VLPs}

Detailed biochemical and biophysical characterization of particles was carried out to ensure their suitability for SVT experiments. One crucial point for these studies is to ensure that the signals observed in microscopic experiments really correspond to single virions rather than to aggregates or particle fractions. This was verified by examining the size distribution of the particle population from the mobility of individual particles in aqueous solution. Trajectories of individual particles with MA.eGFP were extracted from the movies as described in "Materials and methods". The square-displacement of each particle was determined between different consecutive frames and analyzed using the accumulated probability function. The square displacements $r^{2}$ taken from the particle trajectories were sorted by ascending order to form the cumulative probability distribution, $P\left(r^{2}, t\right)$, shown in the upper panel of
Fig. 1b (Schmidt et al. 1995). The cumulative probability distribution was fit with a two-component model (Schütz et al. 1997):

$P\left(r^{2}, t\right)=1-\left[\alpha \mathrm{e}^{\frac{-r^{2}}{\left\langle r_{1}^{2}\right\rangle}}+(1-\alpha) \mathrm{e}^{\frac{-r^{2}}{\left.r_{2}^{2}\right\rangle}}\right]$

where $\alpha$ is the fraction of the first component and $\left\langle r_{i}^{2}\right\rangle$ is the mean square displacement (MSD) of the $i$ th component. A fit of Eq. (1) to the data is shown as a curve in Fig. 1b. The fit yielded $\alpha=0.98$, indicating that the majority of particles have a similar size and that there are no large aggregates in the preparation.

By performing the cumulative probability distribution analysis for different time intervals, the MSD was calculated as a function of lag time, $\Delta t$, as is shown in the lower panel of Fig. 1b. The linearity of the plot indicates the particles are undergoing Brownian diffusion:

$\left\langle r_{1}^{2}\right\rangle=4 D t$.

The slope of the MSD yields a diffusion coefficient. The diffusion coefficient has been determined over a multitude of experiments with different virus preparations and various buffers, all yielding similar results. The average diffusion coefficient is $D=1.78 \pm 0.11 \mu \mathrm{m}^{2} / \mathrm{s}$. From the Einstein-Stokes equation, an effective hydrodynamic radius can be estimated:

$R_{\text {hyd }}=\frac{k T}{6 \pi \eta D}$,

where $k$ is the Boltzmann constant, $T$ is the temperature, and $\eta$ is the viscosity buffer. From the diffusion coefficient, we estimate an average hydrodynamic radius (including the solvation shell) of $\mathrm{R}_{\text {hyd }}=86 \pm 5 \mathrm{~nm}$ or an average diameter of $172 \pm 10 \mathrm{~nm}$ for single VLPs. The actual diameter of the individual VLPs will, of course, vary over more than $10 \mathrm{~nm}$. The average particle size is comparable to the HIV-1 particle diameter of $145 \pm 25 \mathrm{~nm}$ previously determined by cyro-electron microscopy (Briggs et al. 2003), indicating that the virus preparations provide a relatively homogeneous population of complete, nonaggregated single VLPs.

A dual-color HIV-1 derivative, which carries mRFP1 fused to the HIV-1 accessory protein Vpr (Vpr.mRFP) in addition to MA.eGFP, was also developed and characterized (Lampe et al. 2007). These particles are designed to distinguish between complete, dual-colored VLPs and subviral complexes entering the cytoplasm, which should lose most of the MA.eGFP label upon membrane fusion and retain the Vpr.mRFP1 label. For the studies described here, we prepared non-replication competent particles, which can enter the cell efficiently but are unable to undergo reverse transcription due to lack of the LTR 
regions in the viral genome. We verified that the particles displayed the expected protein composition by immunoblotting against various viral proteins as well as against eGFP and mRFP1 as described in Lampe et al. (2007) (data not shown). Particle preparations were further characterized by fluorescence spectroscopy and displayed the fluorescence characteristics of both eGFP and mRFP1 (data not shown). The validity of our approach depends on a high degree of double-labeling that allows differentiation between complete and subviral particles. Due to the flexible architecture of HIV-1, the amounts of Gag and Vpr are expected to vary significantly from particle to particle. Distinguishing between single-labeled and double-labeled particles depends on the sensitivity of the microscope and the background level of the sample. Characterization of virus preparations was performed by microscopy under conditions suitable for live-cell imaging (Lampe et al. 2007 ) and the preparation was found to contain $>90 \%$ of double-labeled VLPs and less than $2 \%$ of the particles were classified as Vpr.mRFP1 only.

\section{HIV-1-cell membrane interactions}

Using both the single and double-labeled virions, we investigated the attachment of HIV-1 derived particles by real-time live-cell microscopy at $37^{\circ} \mathrm{C}$. The data are summarized in Fig. 2 and Table 1. Consistent with our previously published results (Lampe et al. 2007), we observed four different classes of events: (1) random diffusion of double-labeled particles in the extracellular medium, (2) brief contacts of virions with the plasma membrane, (3) the attachment of complete particles to the plasma membrane and (4) intracellular transport of both single-labeled and doubled-labeled particles. For a quantitative analysis of virus-membrane interactions, we focus here on the extracellular events (types 1-3).

Initial experiments were carried out using HeLaP4 cells. As expected, extracellular VLPs (e.g. Fig. 2a, trace a1) displayed diffusion properties comparable to those observed for freely diffusing VLPs in solution. An example of the second type of behavior, a brief touching of the particle to the cell surface followed by dissociation, is shown in Fig. 2a (trace a2). Multiple consecutive contacts of one particle with the plasma membrane, which we had observed frequently for AAV (6), were only occasionally detected in the case of HIV. From the individual trajectories, we analyzed the number of consecutive contacts made by a single particle with the cell membrane. An exponential distribution of contacts was observed with an average of 1.5 touches per particle (Fig. 3), where the average of the distribution is given by the decay constant. For comparison, an average value of 4.4 contacts per particle was observed for AAV.
Approximately one-fifth (18\%) of the VLPs which made contact with HeLaP4 cells were immediately immobilized at the plasma membrane and did not display significant mobility for the remaining observation period (Fig. 2a, trace a3). Particles stuck to the cell membrane were found to be double labeled, indicating that fusion had not occurred. In time-lapse measurements, we observed that many particles stayed immobile over more than $2 \mathrm{~h}$ at $37^{\circ} \mathrm{C}$. We tested whether this immobilization could be inhibited by interfering with the specific interaction of the HIV-1 envelope protein and the cellular receptor or coreceptor. Pre-incubation of host cells with antibodies against CD4 (anti-CD4) to block the interaction of HIV-1 with its primary receptor did not lead to a reduction in the percentage of particles immobilized upon their first contact with the plasma membrane, whereas the same concentration of anti-CD4 is sufficient to block productive infection (data not shown). Similarly, when experiments were performed in the presence of an antagonist of the relevant HIV-1 co-receptor CXCR4 (AMD 3100), roughly the same percentage (19\%) of VLPs became immobilized on the plasma membrane (Table 1). These findings indicate that specific interactions between the VLPs and the cellular receptors and co-receptors are not responsible for the observed immobilization of a large percentage of VLPs upon contact with the plasma membrane.

\section{Rapid immobilization of VLPs depends on heparan sulfate}

Our initial observations suggested that cell surface heparan sulfates play a major role for the rapid immobilization of VLPs on the plasma membrane (Lampe et al. 2007). To investigate the role of heparan sulfate linked proteoglycans (HSPG) for HIV-1 attachment in a quantitative manner, we carried out comparative experiments quantitating VLP binding to HeLaP4 cells treated with heparinase as well as cell lines containing different amounts of CD4 and heparan sulfate on the cell surface. The relative amounts of these factors on the surface of the cell lines used were verified by flow cytometry. There is significant variation between cells within the same population, but the trends for the different cells lines could still be determined. The standard deviations of the mean value for at least three independent experiments are given in Table 1.

Figure $2 \mathrm{~b}$ shows the interaction of VLPs with HeLaP4 cells that have been treated with heparinase. Enzymatic reduction of HSPG on the cell surface, confirmed by flow cytometry measurements, resulted in a dramatic decrease in VLP immobilization, while brief membrane contacts (trace b2) were still observed (Table 1). As the same virus preparation was used for the various experiments and the results were confirmed with different virus preparations, the 
A

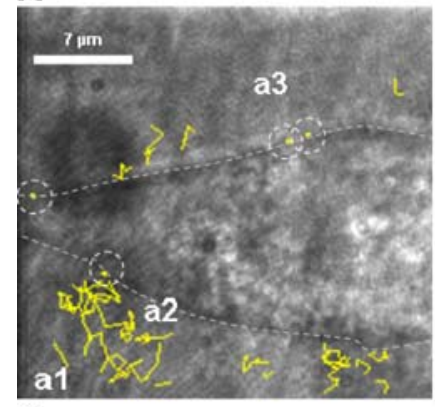

C

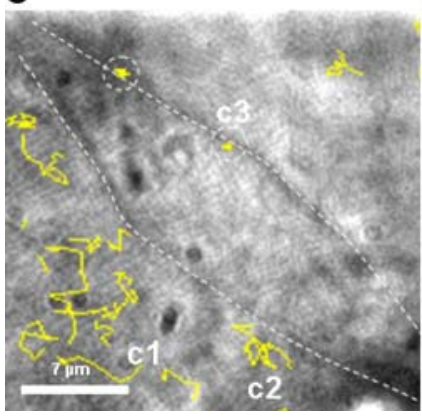

B

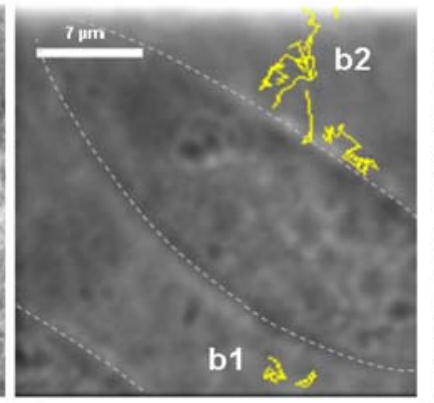

D

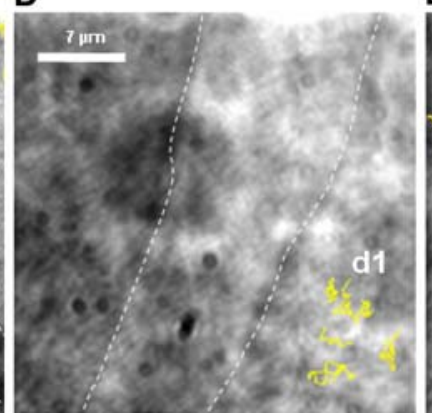

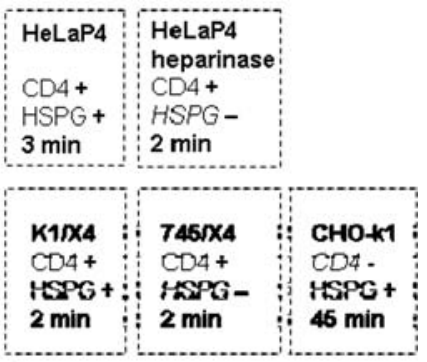

E

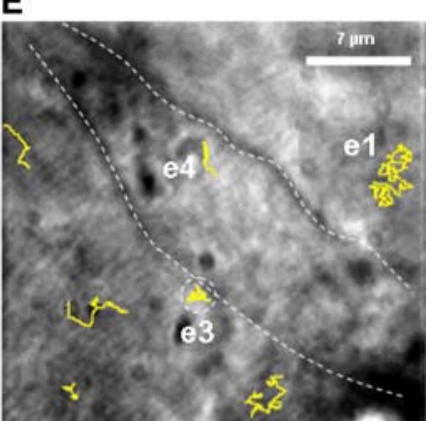

Fig. 2 Interaction of double fluorescent VLPs with HeLaP4 and CHO-derived cells. Trajectories of double-labeled particles are shown in yellow. A bright-field image of the cell is underlaid in grey. a A HeLa P4 cell (CD4+, HSPG+) imaged 3 min after addition of VLPs. Double-labeled VLPs were observed to either undergo free diffusion (a1), make occasional contacts with the plasma membrane (e.g. a2) or to attach to the cell membrane and remain immobile (a3). b A HeLaP4 cell treated with heparinase for $30 \mathrm{~min}$ (CD4+, HSPG-) and subsequently incubated with VLPs for 2 min. Freely diffusing VLPs (b1) and VLPs making brief contacts were observed (b2) but no particles were immobilized on the cell membrane. c A K1/X4 cell
(CD4+, HSPG+) imaged 2 min after VLP addition. The same types of events as for HeLaP4 were observed: freely diffusing VLPs $(c 1)$, particles that make occasional contact with the plasma membrane $(c 2)$ and immobile particles on the cell surface (c3). d A 745/X4 cell (CD4+, HSPG-) imaged 2 min after VLP addition. Freely diffusing VLPs were readily observed $(d l)$. However, there were virtually no particles immobilized at the cell membrane. e A CHO-K1 cell (CD4-, $\mathrm{HSPG}+$ ) imaged $45 \mathrm{~min}$ after VLP addition. Freely diffusing particles $(e 1)$ as well as VLP immobilized in the cell membrane $(e 3)$ were observed. At later time points, double-labeled particles were also detected intracellularly $(e 4)$

Table 1 Correlation between the heparan sulfate concentration on the cell surface and the relative number of immobilized VLPs

\begin{tabular}{|c|c|c|c|c|c|}
\hline Cell lines & Heparan sulfate (FU) & CD4 (FU) & Immobile fraction $(\%)$ & Membrane contacts $\mathrm{N}$ & Contact lifetime $(\mathrm{ms})$ \\
\hline \multicolumn{6}{|l|}{ HeLa derived cell lines } \\
\hline $\mathrm{HeLa}$ & $112 \pm 5$ & $4 \pm 1$ & 1.5 & 794 & $24.4 \pm 1.0$ \\
\hline HeLaP4 & $327 \pm 76$ & $55 \pm 20$ & 18 & 611 & $49 \pm 7$ \\
\hline HeLaP4 (heparinase) & $118 \pm 11$ & $55 \pm 20$ & 2.6 & 289 & $-^{\mathrm{a}}$ \\
\hline HeLaP4 AMD3100 & $327 \pm 76$ & $-{ }^{\mathrm{b}}$ & 19 & 803 & $20.2 \pm 0.9$ \\
\hline HeLaP4 Anti CD4 & $327 \pm 76$ & $-{ }^{\mathrm{b}}$ & 18 & 781 & $23.6 \pm 1.0$ \\
\hline $\mathrm{RC} 25$ & $137 \pm 12$ & $69 \pm 13$ & 2.0 & 697 & $19.9 \pm 1.0$ \\
\hline JC53 & $229 \pm 15$ & $880 \pm 110$ & 5.1 & 1,078 & $19.8 \pm 0.8$ \\
\hline \multicolumn{6}{|l|}{$\mathrm{CHO}$ derived cell lines } \\
\hline pgsA745 & $9 \pm 7$ & $3.2 \pm 0.4$ & 1.4 & 327 & $27.2 \pm 2.1$ \\
\hline $745 / \mathrm{X} 4$ & $7 \pm 1$ & $182 \pm 41$ & 4.0 & 192 & $-{ }^{\mathrm{a}}$ \\
\hline $\mathrm{K} 1$ & $730 \pm 200$ & $3.1 \pm 0.6$ & 19 & 238 & $45 \pm 4$ \\
\hline $\mathrm{K} 1 / \mathrm{X} 4$ & $764 \pm 33$ & $95 \pm 43$ & 33 & 138 & $43 \pm 5$ \\
\hline
\end{tabular}

The relative mean fluorescence intensities from a flow cytometry analysis of cells stained with fluorescently-labeled anti-heparan sulfate or antiCD4 antibodies are shown. The percentage of immobile particles was calculated by normalizing the number of immobilized particles to the sum of particle traces where membrane contact was observed. The last column shows the lifetime for VLPs that dissociated from the cell surface as determined from SVT experiments. The standard error of the fit parameters for the contact lifetimes are given in the table

${ }^{a}$ Histograms for the indicated cell lines could not be satisfactorily described by a single exponential

b The concentration of available CD4 receptors was not determined under these conditions 


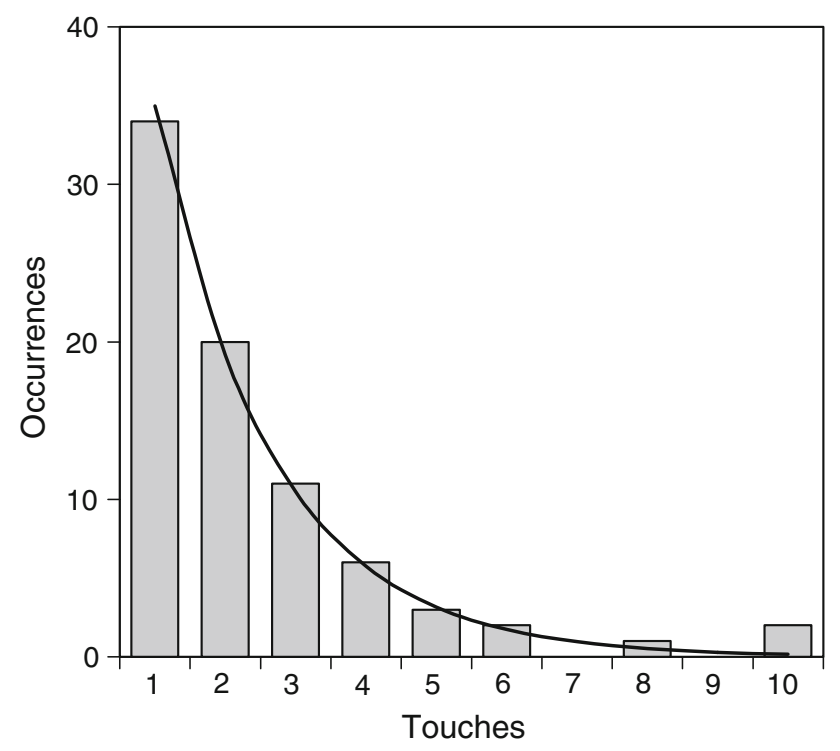

Fig. 3 The histogram of the number of contacts individual VLP make with single HeLaP4 cells. The data are well described by an exponential distribution (solid line). The average number of contacts a VLP makes with the cell is 1.5

percentage of immobilization depends on the properties of the cell surface and is not an artifact from the virus preparation. Furthermore, stable attachment of complete VLPs was recovered upon prolonged incubation $(>1 \mathrm{~h})$, most likely due to newly synthesized HSPG (data not shown).

These results were further supported by using a series of CHO-derived cell lines, which either contained or lacked the HIV-1 receptor (CD4) and the relevant co-receptor (CXCR4), and either contained or lacked HSPG (Zhang et al. 2002). SVT experiments using the HIV-1 infectable $\mathrm{CHO}$ derived cell line $\mathrm{K} 1 / \mathrm{X} 4$ (CD4+/HSPG+), shown in Fig. 2c, yielded similar results as described for HeLaP4 cells (compare with Fig. 2a). Again, occasional brief contacts of extracellular complete particles (trace c2) and immobile double-fluorescent VLPs at the plasma membrane (trace c3) were observed. This result shows that the observed phenotypes are not specific for HeLaP4 cells. SVT experiments with the 745/X4 cell line (CD4+/HSPG-), which lacks HSPG due to a defect in the xylosyl-transferase gene (Zhang et al. 2002), confirmed the role of HSPG in VLP immobilization (Fig. 2d). Freely diffusing extracellular VLPs (trace d1) as well as brief contacts of VLPs with the plasma membrane were readily detected in this case, while virtually no membrane-immobilized particles were found. On the other hand, experiments with CHO-K1 cells (CD4-/HSPG+) again revealed immobilization of complete particles at the plasma membrane (Fig. 2e, trace e3), confirming that Env-CD4 interactions are not required. Intracellular transport of complete VLPs upon prolonged incubation (Fig. 2e, trace e4) in the absence of CD4 presumably reflects the non-specific uptake of particles via endocytosis. To further confirm that the amount of CD4 on the cell surface is not responsible for the observed immobilization, we also compared VLP binding to HeLa derivatives, which had been selected for high (JC-53) and low (RC-25) expression of CD4 (Platt et al. 1998). Both cell lines showed greatly reduced VLP binding as compared to HeLaP4, although the amount of CD4 was similar to HeLaP4 on RC-25 and much higher on JC-53 cells (Table 1). The surface concentrations of HSPG in both RC-25 and JC-53 cell lines are significantly lower than that of HeLaP4 (Table 1). Hence, the reduced binding is consistent with our observation that HSPG immobilizes VLPs on the cell surface.

To complement and validate these live-cell imaging studies by a more traditional approach, we investigated the correlation between HSPG and CD4 concentration, respectively, and the amount of VLPs bound to the cell using flow cytometry. Cells were incubated with fluorescent VLPs on ice to prevent internalization of particles bound to the cell membrane. After washing away unbound particles, the amount of cell-associated eGFP fluorescence was quantitated by flow cytometry and used as a measure for the number of immobilized VLPs. The results were plotted against the CD4 or heparan sulfate concentrations on the cell surface (Fig. 4a, c respectively). As indicated by the horizontal error bars, the cell lines showed variation in HSPG content dependent on the passage of the cells used for analysis. Nevertheless, a clear linear correlation was observed between the number of bound VLPs and the cell surface concentration of heparan sulfate. As shown in Fig. 4, the results obtained in these flow cytometry experiments corroborated the results obtained by SVT analyses. The fraction of immobilized particles relative to the total number of particles that made contact with the cells showed a linear correlation with HSPG concentration (Fig. 4b). In contrast, there was no correlation between VLP binding and cell surface CD4 concentration detected in either assay (Fig. 4c, d; Table 1). These results provide clear evidence that rapid and prolonged immobilization of HIV-1 derived particles is mediated by stable attachment of HIV-1 to heparan sulfate. HSPG is clearly not required for HIV-1 infection of the target cells used in this study, and heparinase treatment caused only a mild reduction in infectivity (Lampe et al. 2007). However, the effect of HSPG on HIV infectivity may vary depending on the properties of the viral envelope (Zhang et al. 2002) and possibly also on the host cell.

Contact duration

While approximately $20 \%$ of VLPs that made contact with the plasma membrane of HeLaP4 cells became immobilized 

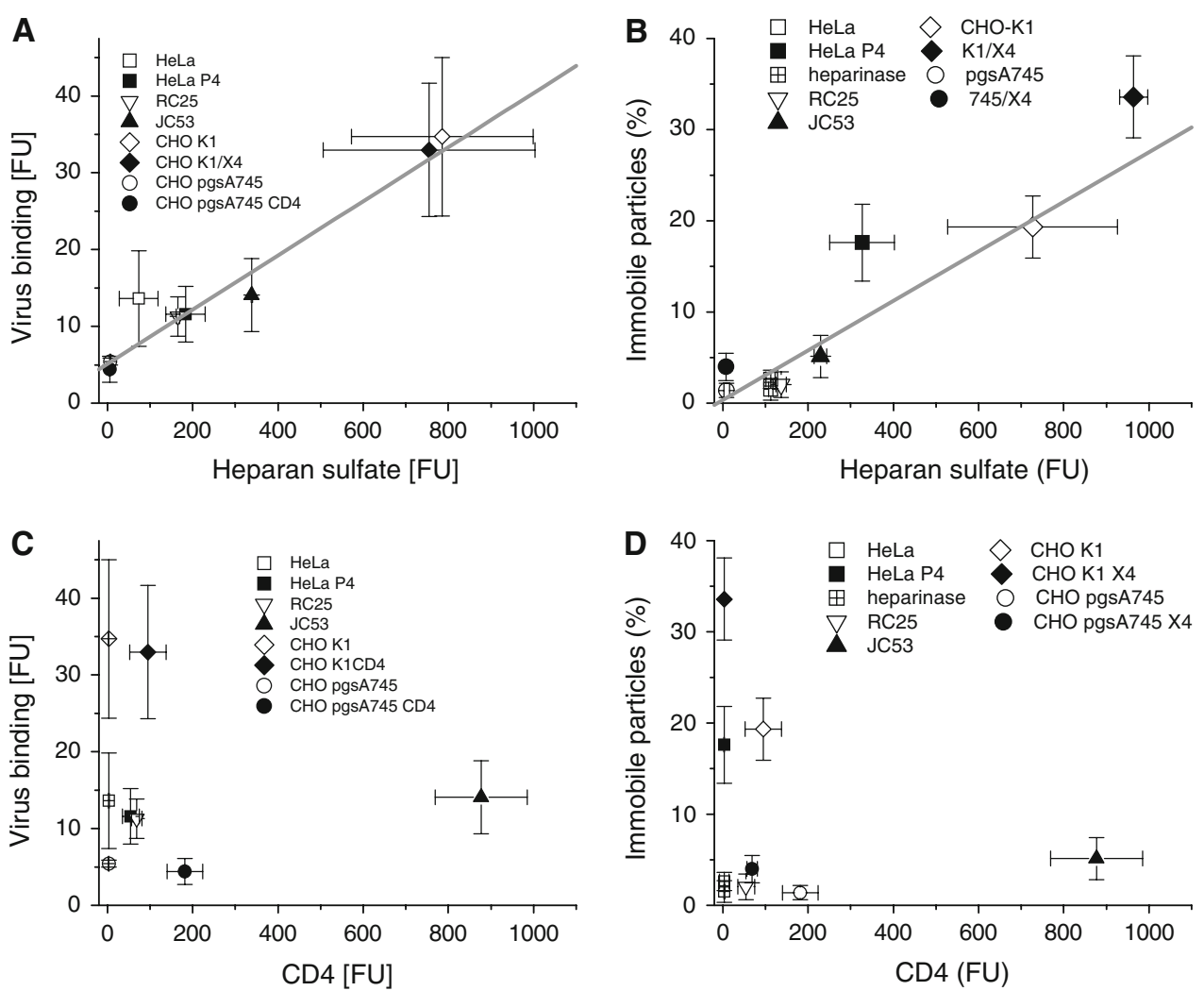

Fig. 4 Correlation between VLP binding and cell-surface concentration of heparan sulfate and CD4. a The indicated cell lines were incubated with labeled VLPs for $30 \mathrm{~min}$ on ice and virus binding was quantified by flow cytometry analysis (in arbitrary fluorescence units FU). Virus binding was plotted against cell surface heparan sulfate concentration, determined in parallel by flow cytometry analysis using a heparan sulfate specific antibody. The error bars represent the standard deviation of at least three independent measurements. b The number of VLPs immobilized in SVT experiments at $37^{\circ} \mathrm{C}$ (normalized for the number of total cell contacts evaluated) plotted against

the cell surface concentration of heparan sulfate of the indicated cell lines as determined by flow cytometry. Lines represent the best linear fit to the data. The vertical error bars represent the standard deviation in particle number assuming Poissonian statistics and the horizontal error bars, as above, represent the standard deviation from flow cytometry measurements. $\mathbf{c}$ The correlation of VLPs bound to the cell surface compared to CD4 concentration. Experiments were performed as in (a) with the alteration that a fluorescently labeled CD4 specific antibody was used to quantify the CD4 concentration. d The same data as in (b) plotted versus CD4 concentration

immediately, the remaining $80 \%$ made only a transient contact with the cell surface. The time scale of the VLP-cell surface interactions were derived from the trajectories of individual viruses as described in "Materials and methods". We analyzed the contact behavior of the $80 \%$ of VLPs that made transient interactions with the plasma membrane. A histogram of contact times for VLPs interactions with various cell lines are shown in Fig. 5. We observed that greater than $94 \%$ of the transient contacts lasted less than $240 \mathrm{~ms}$. Therefore, the overall distribution is bimodal, with the vast majority of virions either being immobilized via interaction with HSPG or dissociating from the cell surface within $240 \mathrm{~ms}$. These short interaction times with the cell surface may allow viruses to come in contact with many different parts of surrounding cells in their search for a receptor. There are very few dissociation events detected in the intermediate time scale of $240 \mathrm{~ms}-16 \mathrm{~s}$. As the infection

rate of HIV-1 is low, we cannot presently determine whether those interactions of intermediate duration are relevant for fusion.

Single virus tracing allows us to investigate details of the short interaction between VLPs and the cell surface. The histograms of contact times for HeLa and HeLaP4 cells are shown in Fig. 5a, b respectively (Table 1). The distributions can be described by a single exponential decay with lifetimes of 24 and 49 ms respectively. As an exponential distribution has a well-defined statistics that stretches over several frames, the lifetime can be determined with much higher resolution than the temporal resolution of the measurement. Blocking interactions between VLPs and CD4 by preincubation of HeLaP4 cells with anti-CD4 antibody resulted in contact times identical within error to parental HeLa cells, which do not express the CD4 receptor (Fig. 5c). While we have found no 
Fig. 5 Duration of VLP contacts with the plasma membrane for HeLa, HeLaP4 and $\mathrm{CHO}$ cells. The histogram of VLP-cellular interaction times determined from SVT measurements are shown. Single exponential decays that have either been fit to the data (solid line) or taken from other fits (dotted line) are displayed in each panel. a HeLa cells ( $\tau=24 \mathrm{~ms})$, b HeLaP4 cells ( $\tau=49 \mathrm{~ms})$, c HeLaP4 cells that have been preincubated with anti-CD4 ( $\tau=24 \mathrm{~ms})$, d HeLaP4 cells that have been pretreated with heparinase. The data cannot be described with a single exponential function; for comparison, the single exponential decay from panel $C$ with $\tau=24 \mathrm{~ms}$ is shown, e CHO pgsA745 cells ( $\tau=27 \mathrm{~ms}$ ), and f CHO 745 $\mathrm{X} 4$ cells. The data cannot be described with a single exponential function; for comparison, the single exponential decay from panel $E$ with $\tau=27 \mathrm{~ms}$ is shown
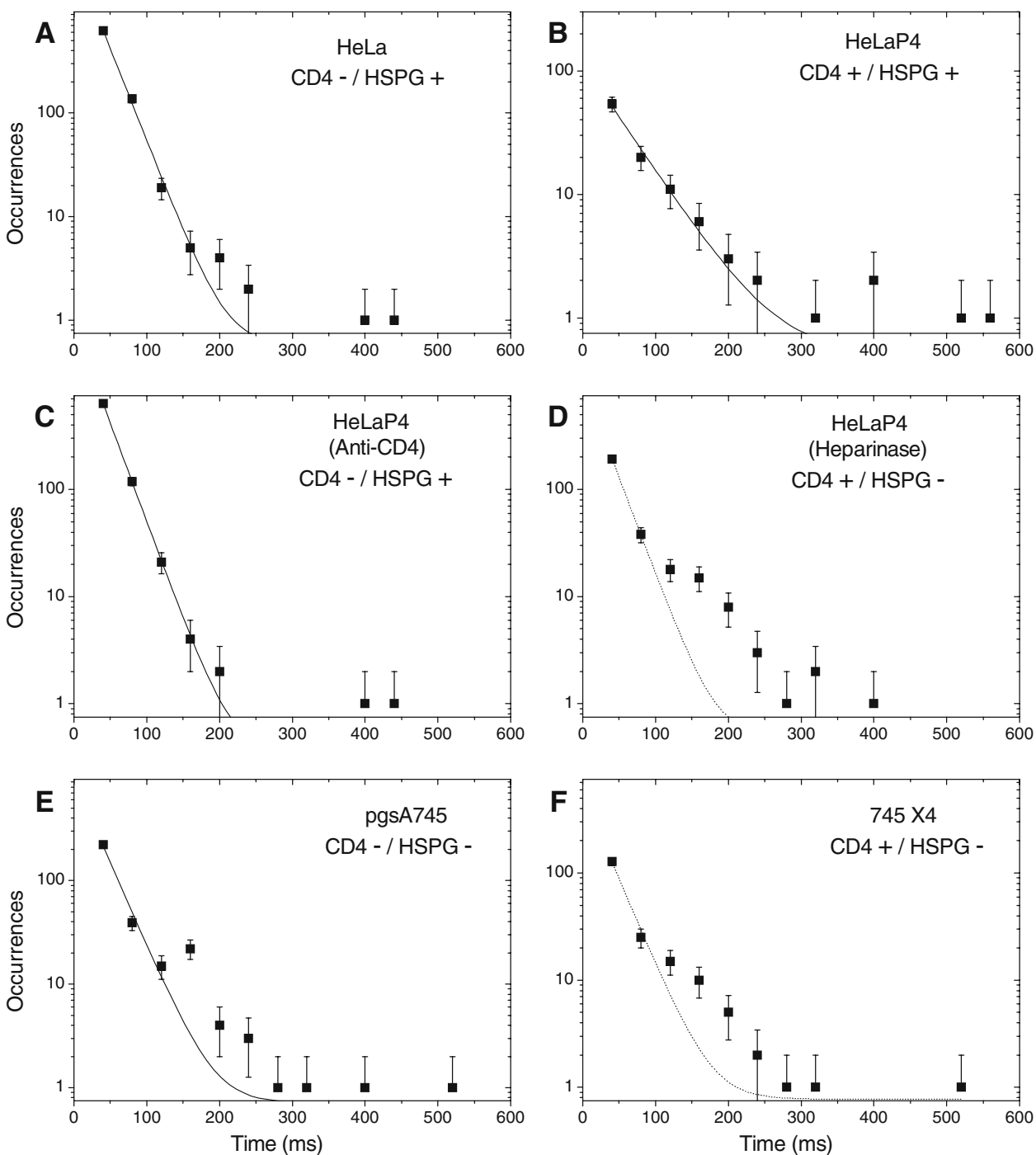

correlation between the amount of CD4 and the number of VLPs immobilized on the cell surface, weak non-productive interactions with CD4 may lead to the observed prolongation of transient contacts between the virus and the cell in the presence of CD4. In agreement with this hypothesis, a shoulder in the histogram of contact times at $160 \mathrm{~ms}$ is apparent in the absence of HSPGs (Fig. 5d-f), suggesting slightly more stable transient contacts between the virus and surface molecules other than HSPGs. In measurements with HSPG positive cell lines, this shoulder was not apparent due to the much larger relative proportion of brief contacts made with HSPG. The shoulder is more prominent when CD4 was present (Fig. 5d, f), hinting that CD4 may be responsible for the increased duration of the virus-cell interaction. However, the fact that a weak shoulder is also visible in CD4 negative cells (Fig. 5e) suggests that other factors may also be important. If the shoulder is due to CD4-VLP interactions, a high concentration of CD4 should lead to the observation of a shoulder in the contact time distribution, even in the presence of HSPG. To investigate this possibility, further experiments were therefore performed using JC53 cells. JC53 cells display an approximately 10-fold higher concentration of CD4 present on the cell surface. The results showed a single exponential decay with lifetime of $\sim 20 \mathrm{~ms}$ (data not shown), suggesting that the situation is more complex with no simple correlation between the contact lifetime and the concentration of CD4. Using SVT, a comprehensive analysis of the biomolecules involved in the HIV-cell surface interactions is now possible, but is beyond the scope of this publication.

\section{Conclusion}

Using SVT, we have investigated the interaction of viruslike particles (VLPs) with the cell surface. Fluorescent protein-labeled HIV-1 particles were prepared as described 
previously (Lampe et al. 2007). From measurements on freely diffusing VLPs, the virus preparation was determined to be homogenous and the size of the particles corresponded to that of single viruses with a hydrodynamic radius of $86 \pm 5 \mathrm{~nm}$. The VLPs showed two types of interactions with the plasma membrane: brief interactions with the cell surface where the VLP rapidly dissociates and diffused away or immobilization. Approximately $20 \%$ of the VLPs (in HeLaP4 cells) that contacted the plasma membrane became immediately immobilized on the cell surface. This behavior correlates with the surface concentration of HSPG on the target cells as shown with flow cytometry and SVT experiments. The majority of VLPs that are not immobilized only interact briefly with the cell surface. The contact duration follows an exponential distribution with a lifetime of $20-50 \mathrm{~ms}$ in the cell lines studied here. Hence, the dominant dynamics of HIV-cell interaction are very rapid. Either the virion becomes immobilized upon the first binding event, or it interacts for a few milliseconds and dissociates. This short duration of transient contacts defines a very limited time frame for the virus to probe the cell surface for its cognate receptor. Thus, cytoplasmic entry may be stochastic, its occurrence depending on whether the initial contact was made within an area of high receptor density. Prolonged cell attachment mediated by HSPG could theoretically improve the likelihood of contacting receptor molecules, but may not allow sufficient motility to probe larger surface areas in the case of HIV-1. Stronger beneficial effects of HSPG as an attachment factor have been reported for other viruses, e.g. herpesviruses, reviewed in (Rostand and Esko 1997).

\begin{abstract}
Acknowledgments We thank Maria Anders and Monika Franke for expert technical assistance. We gratefully acknowledge the generous gift of the plasmids for peGFP-Vpr by Tom Hope (University of Illinois, Chicago), pRSET-mRFP1 by Roger Tsien (University of California, San Diego) and pMM310 by Ned Landau (Salk Institute, La Jolla), the gift of cell lines CHO-K1, pgsA745, K1/X1 and 745/X1 by Paul Bieniasz (Aaron-Diamond-Centre, New York) and of antiserum against gp120 by Valerie Bosch (DKFZ Heidelberg). We thank W. Muranyi, S. Mugrauer, C. Kofink and M. Göbel for experimental support and S. Ivanchenko for assistance with the lifetime analysis. This work was supported by the Deutsche Forschungsgemeinschaft (grant KR906/4-1 and MU 885/4-2 to B. M and H. G. K and BR1027/ 16-2 to C. B), the Schwerpunktprogramm 1175 (D. C. L and C. B, H. G. K), the Ministry of Science, Research and the Arts of BadenWürttemberg (23-7532.24-22-21-12/1 to B. M and H. G. K) and the German Excellence Initiative via the "Nanosystems Initiative Munich (NIM)" (D. C. L and C. B). J. A. G. B was supported by fellowships from the Alexander von Humboldt Foundation and EMBO.
\end{abstract}

Open Access This article is distributed under the terms of the Creative Commons Attribution Noncommercial License which permits any noncommercial use, distribution, and reproduction in any medium, provided the original author(s) and source are credited.

\section{References}

Bobardt MD, Saphire AC, Hung HC, Yu X, Van der Schueren B, Zhang Z, David G, Gallay PA (2003) Syndecan captures, protects, and transmits HIV to T lymphocytes. Immunity 18:2739

Bräuchle C, Seisenberger G, Endress T, Ried MU, Büning H, Hallek M (2002) Single virus tracing: visualization of the infection pathway of a virus into a living cell. Chemphyschem 3:299-303

Briggs JA, Wilk T, Welker R, Kräusslich HG, Fuller SD (2003) Structural organization of authentic, mature HIV-1 virions and cores. EMBO J 22:1707-1715

Charneau P, Mirambeau G, Roux P, Paulous S, Buc H, Clavel F (1994) HIV-1 reverse transcription. A termination step at the center of the genome. J Mol Biol 241:651-662

Daecke J, Fackler OT, Dittmar MT, Kräusslich HG (2005) Involvement of clathrin-mediated endocytosis in human immunodeficiency virus type 1 entry. J Virol 79:1581-1594

Damm EM, Pelkmans L, Kartenbeck J, Mezzacasa A, Kurzchalia T, Helenius A (2005) Clathrin- and caveolin-1-independent endocytosis: entry of simian virus 40 into cells devoid of caveolae. J Cell Biol 168:477-488

Ewers H, Smith AE, Sbalzarini IF, Lilie H, Koumoutsakos P, Helenius A (2005) Single-particle tracking of murine polyoma virus-like particles on live cells and artificial membranes. Proc Natl Acad Sci USA 102:15110-15115

Fackler OT, Peterlin BM (2000) Endocytic entry of HIV-1. Curr Biol 10:1005-1008

Finke S, Brzozka K, Conzelmann KK (2004) Tracking fluorescencelabeled rabies virus: Enhanced green fluorescent protein-tagged phosphoprotein $\mathrm{P}$ supports virus gene expression and formation of infectious particles. Journal of Virology 78:12333-12343

Lakadamyali M, Rust MJ, Babcock HP, Zhuang X (2003) Visualizing infection of individual influenza viruses. Proc Natl Acad Sci USA 100:9280-9285

Lampe M, Briggs JAG, Endress T, Glass B, Riegelsberger S, Kräusslich H-G, Lamb DC, Bräuchle C, Müller B (2007) Double-labeled HIV-1 particles for study of virus-cell interaction. Virology 360:92-104

Lehmann MJ, Sherer NM, Marks CB, Pypaert M, Mothes W (2005) Actin- and myosin-driven movement of viruses along filopodia precedes their entry into cells. J Cell Biol 170:317-325

Maurin T, Fenard D, Lambeau G, Doglio A (2007) An envelopedetermined endocytic route of viral entry allows HIV-1 to escape from secreted phospholipase A2 entry blockade. J Mol Biol 367:702-714

McDonald D, Vodicka MA, Lucero G, Svitkina TM, Borisy GG, Emerman M, Hope TJ (2002) Visualization of the intracellular behavior of HIV in living cells. J Cell Biol 159:441-452

Moulard M, Lortat-Jacob H, Mondor I, Roca G, Wyatt R, Sodroski J, Zhao L, Olson W, Kwong PD, Sattentau QJ (2000) Selective interactions of polyanions with basic surfaces on human immunodeficiency virus type 1 gp120. J Virol 74:1948-1960

Müller B, Daecke J, Fackler OT, Dittmar MT, Zentgraf H, Kräusslich HG (2004) Construction and characterization of a fluorescently labeled infectious human immunodeficiency virus type 1 derivative. J Virol 78:10803-10813

Parren PWHI, Mondor I, Naniche D, Ditzel HJ, Klasse PJ, Burton DR, Sattentau QJ (1998) Neutralization of human immunodeficiency virus type 1 by antibody to gp120 is determined primarily by occupancy of sites on the virion irrespective of epitope specificity. J Virol 72:3512-3519

Platt EJ, Wehrly K, Kuhmann SE, Chesebro B, Kabat D (1998) Effects of CCR5 and CD4 cell surface concentrations on 
infections by macrophagetropic isolates of human immunodeficiency virus type 1. J Virol 72:2855-2864

Rostand KS, Esko JD (1997) Microbial adherence to and invasion through proteoglycans. Infect Immun 65:1-8

Rust MJ, Lakadamyali M, Zhang F, Zhuang X (2004) Assembly of endocytic machinery around individual influenza viruses during viral entry. Nat Struct Mol Biol 11:567-573

Schmidt T, Schütz GJ, Baumgartner W, Gruber HJ, Schindler H (1995) Characterization of photophysics and mobility of single molecules in a fluid lipid membrane. J Phys Chem A 99:1766217668

Schütz GJ, Schindler H, Schmidt T (1997) Single-molecule microscopy on model membranes reveals anomalous diffusion. Biophys J 73:1073-1080
Seisenberger G, Ried MU, Endress T, Büning H, Hallek M, Bräuchle C (2001) Real-time single-molecule imaging of the infection pathway of an adeno-associated virus. Science 294:1929-1932

Suomalainen M, Nakano MY, Keller S, Boucke K, Stidwill RP, Greber UF (1999) Microtubule-dependent plus- and minus enddirected motilities are competing processes for nuclear targeting of adenovirus. J Cell Biol 144:657-672

Ugolini S, Mondor I, Sattentau QJ (1999) HIV-1 attachment: another look. Trends Microbiol 7:144-149

Zhang YJ, Hatziioannou T, Zang T, Braaten D, Luban J, Goff SP, Bieniasz PD (2002) Envelope-dependent, cyclophilin-independent effects of glycosaminoglycans on human immunodeficiency virus type 1 attachment and infection. J Virol 76:6332-6343 